\title{
MEASURABILITY OF LATTICE OPERATIONS IN A CONE
}

\author{
KOHUR GOWRISANKARAN
}

\begin{abstract}
Let $X$ be a locally convex Hausdorff topological vector space and $C$ a convex cone generating $X$ such that $C$ is a lattice in its own order. Under suitable conditions $(x, y) \rightarrow \sup (x, y)$ and $\inf (x, y)$ are shown to be measurable mappings.
\end{abstract}

Let $X$ be a locally convex Hausdorff topological vector space over the real numbers. Let $C$ be a closed proper convex cone with vertex 0 and let $C$ generate $X$. Further, let $C$ be a lattice in its own order. There are wellknown results asserting the continuity of the mappings $(x, y) \rightarrow \sup (x, y)$ and $(x, y) \rightarrow \inf (x, y)$ under suitable restrictions on the cone $C([\mathbf{4}$, Chapter V], [2, Appendix]). In this note we shall give conditions under which the lattice operations are measurable mappings. This measurability was found to be very useful in our recent work in potential theory [1].

THEOREM 1. Let $X$ be a Hausdorff locally convex real topological vector space. Let $C$ be a closed proper convex cone with vertex at the origin, generating $X$ and such that $C$ is a lattice in its own order. Let $B$ be a compact metrizable base for $C$.

Then, the mappings: $C \times C \rightarrow C$ given by $(x, y) \rightarrow \sup (x, y)$ and $(x, y) \rightarrow$ $\inf (x, y)$ are Borel, viz., the inverse image of any Borel set of $C$ under each of these mappings is a Borel set of $C \times C$.

Proof. Step (1). Let us denote by $K$ the set of all positive continuous linear functionals on $X$, and $Y=K-K$ the vector space generated by this cone. We note that $Y$ separates the points of $X$ [4, Example 25, p. 71] and hence $\sigma(x, y)$ on $X$ is a Hausdorff topology. Let us denote by $\sigma$ the topology induced on $C$ by $\sigma(x, y)$ on $X$. Let $\tau$ be the given topology on $X$. We note that $(C, \tau)$ is locally compact, metrizable and separable and hence it is a polish space. It follows that the $(C, \sigma)$ Borel sets and the $(C, \tau)$ Borel sets are identical and the same is the case on the product space $C \times C$ with the respective product topologies [5].

Presented to the Society, September 18, 1972; received by the editors September 11, 1972 and, in revised form, March 12, 1973.

AMS (MOS) subject classifications (1970). Primary 46A40; Secondary 28A20, 31C05, $31 \mathrm{D} 05$.

Key words and phrases. Ordered vector space, cone, lattice, compact base, Borel function.

(e) American Mathematical Society 1973 
Step (2). Let $L_{0}$ be the positive continuous linear functional on $X$ defining the base $B$, i.e., $B=\left\{x \in C: L_{0}(x)=1\right\}$ [3, Theorem 3.6]. Let $x$ and $y$ be any two elements of $C$ and $L_{0}(x+y)=a$. The set of all elements $z$ in $C$ majorizing both $x$ and $y$ and such that $z \leqq x+y$ is decreasingly directed and is contained in the $\tau$-compact set $\left\{z \in C: L_{0}(z) \leqq a\right\}$. Therefore, it is possible to choose a decreasing sequence $\left\{z_{n}\right\}$ satisfying $(i) z_{n} \leqq x+y$ for every $n$, and (ii) $\left\{z_{n}\right\} \tau$-converges to $\sup (x, y)$.

Step (3). Let us fix a positive continuous linear functional $L$ on $X$. We shall show that $(x, y) \rightarrow L[\sup (x, y)](\operatorname{resp} .(x, y) \rightarrow L[\inf (x, y)])$ is a lower (resp. upper) semicontinuous function on $C \times C$. It is sufficient to prove the lower semicontinuity of the first mapping. For this, consider $\left(x_{n}, y_{n}\right)$ in $C \times C$ converging to $\left(x_{0}, y_{0}\right)$ such that $x_{n}, y_{n}$ for all $n$ and $x_{0}, y_{0}$ belong to a fixed compact set $A$ of $C, A$ of the form $\left\{z: L_{0}(z) \leqq b\right\}$. Given $\varepsilon>0$, it is possible to choose an element $s_{n}$, for every $n$, such that (i) $s_{n}$ belongs to $A+A$, (ii) $s_{n}$ majorizes both $x_{n}$ and $y_{n}$ and (iii) $L\left[\sup \left(x_{n}, y_{n}\right)\right]>$ $L\left(s_{n}\right)-\varepsilon / 2$. Hence, given any subsequence, $\left\{n \in M^{\prime} \subset N\right\}$, we may choose a further subsequence, say $\left\{n \in M \subset M^{\prime} \subset N\right\}$, such that as $n$ in $M$ tends to infinity, $\left\{s_{n}\right\}$ converges to an element $z$ in $C$. Hence, for all sufficiently large $n$ in $M, L\left(s_{n}\right)>L(z)-\varepsilon / 2$ and consequently, $L\left[\sup \left(x_{n}, y_{n}\right)\right]>$ $L(z)-\varepsilon$.

We now claim that $z$ is a majorant of $\sup \left(x_{0}, y_{0}\right)$. Since $s_{n}$ is a majorant of both $x_{n}$ and $y_{n}$, there are elements $t_{n}$ and $u_{n}$ in $C$ such that $s_{n}=x_{n}+t_{n}$ and $s_{n}=y_{n}+u_{n}$ for every $n$. It is clear that $\left\{t_{n}\right\}$ and $\left\{u_{n}\right\}$ converge respectively to $t_{0}$ and $u_{0}$ in $C$ as $n$ in $M$ tends to infinity. Hence, $z=x_{0}+t_{0}$ and $z=y_{0}+u_{0}$ proving the assertion $z \geqq \sup \left(x_{0}, y_{0}\right)$. We deduce from the above inequalities that for all sufficiently large $n$ in $M$,

$$
L\left[\sup \left(x_{n}, y_{n}\right)\right]>L(z)-\varepsilon \geqq L\left[\sup \left(x_{0}, y_{0}\right)\right]-\varepsilon .
$$

This proves the lower semicontinuity of the function $(x, y) \rightarrow L[\sup (x, y)]$.

Step (4). Define for any $L$ in $K$ and nonnegative real numbers $a$ and $b$, $W(L, a, b)=\{x \in C: a<L(x)<b\}$. The sets of the form $W(L, a, b)$ constitute a subbasis for the open sets of $(C, \sigma)$. However, $(C, \sigma)$ is a strongly Lindelöf space, i.e., every open subspace is Lindelöf. Hence, each $\tau^{\prime}$ open set in $C$ is a countable union of finite intersections of sets of the form $W(L, a, b)$. Hence, the sets of the form $W(L, a, b)$ generate the Borel $\sigma$-algebra of $(C, \sigma)$. But

$$
\begin{aligned}
\{(x, y) \in C \times C: \sup (x, y) \in W(L, a, b)\} & \\
& =\{(x, y) \in C \times C: a<L[\sup (x, y)]<b\}
\end{aligned}
$$

is a Borel subset of $C \times C$, proving the Borel measurability of $(x, y) \rightarrow$ $\sup (x, y): C \times C \rightarrow C$. The Borel measurability of the infimum is deduced 
in a similar way from the upper semicontinuity of $(x, y) \rightarrow L[\inf (x, y)]$. The theorem is proved.

EXAmPLE. Let $\mathrm{H}^{+}$be the set of all positive harmonic functions on the unit disc $U$ and let $H$ be the differences of elements in $H^{+}$. Then, $H$ provided with the topology of uniform convergence on compact subsets of $U$ is a locally convex space and it is well known that $H$ and $H^{+}$satisfy all the conditions of the above theorem. We shall show that the lattice operations are not continuous on $H^{+}$. Let $P=e^{i \theta}$ and $P_{n}=e^{i \theta_{n}}$ be points on the unit circle such that $P_{n}$ converges to $P$ and $P_{n} \neq P_{m}$ if $n \neq m$. Let $u_{n}(r, \varnothing)$ be the Poisson function with pole at $\theta_{n}$, i.e.,

$$
u_{n}(r, \varnothing)=\left(1-r^{2}\right) /\left[1-2 r \cos \left(\theta_{n}-\varnothing\right)+r^{2}\right] \text {. }
$$

Then $u_{n}$ belongs to $H^{+}$, and it is clear that $u_{n}$ converges to $u$, the Poisson function with pole at $e^{i \theta}$. Since $u_{n}$ is an extreme element of the base $B=\left\{w \in H^{+}: w(0)=1\right\}$ (and so is $u$ ), it is easy to see that $\sup \left(u_{n}, u\right)=u_{n}+u$ and hence $\sup \left(u_{n}, u\right)$ converges to $2 u$ which is not equal to $u=$ $\sup \left(\lim u_{n}, u\right)$.

It is natural to raise the question whether the various mappings $x \rightarrow x^{+}$, $x \rightarrow x^{-}, \quad(x, y) \rightarrow \sup (x, y)$ and $(x, y) \rightarrow \inf (x, y)$ are measurable. It is easily verified that the measurability of one of them is equivalent to the measurability of all the mappings. Now, we have the following result.

THEOREM 2. Let $X$ and $C$ be as in the earlier theorem. Then the mapping $x \rightarrow x^{+}$is $\mu$-Lusin measurable for every locally finite Borel measure $\mu$ on $X$.

Proof. We observe that $X$ is a Suslin space, since it is the image of the polish space $C \times C$ under the continuous mapping $(x, y) \rightarrow x-y$. Hence, there is a mapping $\varphi: X \rightarrow C \times C$ such that $\varphi$ composed with $(x, y) \rightarrow x-y$ is identity on $X$ and $\varphi$ is $\mu$-Lusin measurable for every Radon measure $\mu$ on $X$ [5].

Let now $K$ be a compact set of $X$ and $\mu$ a locally finite Borel measure on $X$. Then $\mu$ is a Radon measure [5]. Given $\varepsilon>0$, there exists a compact set $K_{\varepsilon} \subset K$ such that $\mu\left(K_{\varepsilon}\right)>\mu(K)-\varepsilon$ and $\varphi$ restricted to $K_{\varepsilon}$ is continuous. Hence, $\varphi$ restricted to $K_{\varepsilon}$ is a homeomorphism onto $\varphi\left(K_{\varepsilon}\right)$. From this we deduce that $\left\{|x|: x \in K_{\varepsilon}\right\}$ is contained in a compact set of $C$. Also, since $K_{\varepsilon}$ is compact and Suslin it is a metrizable space. Now, exactly as in the proof of Theorem 1 , it can be shown that $x \rightarrow x^{+}: K_{\varepsilon} \rightarrow C$ is Borel measurable. But $C$ being a polish space, we deduce that $x \rightarrow x^{+}$is, in fact, a $\mu$-Lusin measurable function on $X$. The proof is complete.

Remark. For $X$ and $C$ as above, it can be shown that there is always a point of discontinuity of the mapping $x \rightarrow x^{+}$. 


\section{BIBLIOGRAPHY}

1. K. Gowrisankaran, Integral representation for a class of multiply superharmonic functions, Ann. Inst. Fourier (Grenoble) 23 (1973), fasc. 4.

2. J. L. Kelley, I. Namioka, et al., Linear topological spaces, University Series in Higher Math., Van Nostrand, Princeton, N.J., 1963. MR 29 \#3851.

3. A. L. Peressini, Ordered topological vector spaces, Harper \& Row, New York, 1967. MR 37 \#3315.

4. H. H. Schaefer, Topological vector spaces, Macmillan, New York, 1966. MR 33 \#1689.

5. L. Schwartz, Radon measures on general topological spaces, Tata Institute of Fundamental Research Monographs (to appear).

Department of Mathematics, McGill University, Montreal, Quebec, Canada 